\title{
Joint Optimization of Precoder and Decoder in Multiuser MIMO Systems
}

\author{
M. Raja ${ }^{1}$, Ha H. Nguyen ${ }^{2}$, P. Muthuchidambaranathan ${ }^{1}$ \\ ${ }^{1}$ Department of Electronics and Communication Engineering, National Institute of Technology (NIT), \\ Tiruchirappalli, India. \\ 2 Department of Electrical \& Computer Engineering, University of Saskatchewan, 57 Campus Dr., \\ Saskatoon, Saskatchewan, Canada, S7N 5A9.
}

Correspondence: Ha H. Nguyen, ha.nguyen@usask.ca

Manuscript communication: received 20 April 2012, accepted 26 May 2012

\begin{abstract}
This paper considers the joint optimization of precoder and decoder for both uplink and downlink transmissions in multiuser multiple-input, multiple-output (MU-MIMO) systems. Focusing on the scenario when an improper constellation such as binary phase shift-keying (BPSK) or $M$-ary amplitude shift-keying ( $M$-ASK) is employed, novel joint linear precoders and decoders are proposed to minimize the total mean squared error (TMSE) of the symbol estimation. The superiority of the proposed transceivers over the previously-proposed designs is thoroughly verified by simulation results.
\end{abstract}

Keywords- Multi-user MIMO system, transceiver design, linear precoding, linear decoding, improper constellations.

Multiuser MIMO (MU-MIMO) wireless communications systems have received a great attention in recent years due mainly to their ability to provide improvements in capacity and reliability of information transmission over wireless channels [1], [2]. Various performance measurements have been considered to design joint transceiver structures for MU-MIMO systems with both uplink and downlink configurations, such as minimum mean-square error (MMSE) from all the data streams, maximum sum capacity and minimum bit error rate (BER) [3], [4].

A joint linear transceiver design for uplink MU-MIMO systems with minimum total mean-square error (TMSE) has been investigated in [5]. On the other hand, a joint linear transceiver design for downlink MU-MIMO systems is discussed in [6]-[8] under the assumption of perfect channel state information (CSI) at both the transmitter and receiver. To enable precoding at the transmitter, channel estimation has to be performed at the receiver and fed back to the transmitter [9]. But the feedback information is generally not perfect because of the channel estimation error and/or delay. In [10][12], imperfect CSI is considered and joint transceiver designs for TMSE minimization are formulated for both uplink and downlink transmissions.

Specifically, the minimum TMSE designs in [10]-[12] are formulated as nonconvex optimization problems under a total transmit power constraint. The linear transceivers for uplink and downlink MU-MIMO systems are obtained with iterative algorithms. In [10], [11], the imperfect CSI is taken into account by including the effect of channel estimation error. The channel correlation (at the transmitter for uplink, and at the receiver for downlink) is considered [10], [11], whereas [13], [14] examine both transmit and receive correlations. The feedback information is assumed to be error-free for the ease of analysis. In [15], both linear and non-linear transceiver structures for flat fading MIMO channels have been studied under the assumption that the CSI is available at the transmitter. In general, the linear structure is mostly preferred in transceiver design due to its lower computation complexity as compared to non-linear designs.

Reference [16] proposes novel linear precoding schemes for single-user and multiuser MIMO systems that perform better than existing linear precoders when the so-called improper modulation schemes are used. Such performance improvement is obtained by working with the modified cost functions that take into account the properties of improper signal constellations. The designs are considered for both perfect and imperfect CSI.

More recently, our work in [17] studies a joint linear transceiver (precoder and decoder) design that minimizes the TMSE of the symbol estimation at the output of the decoder for SU-MIMO systems with improper modulation. The work considers both the scenarios of perfect and imperfect CSI. This paper is a further development of [17] to the MU-MIMO systems that employ improper modulation. The joint linear precoding/decoding designs are presented for both downlink and uplink transmissions. The presentation focuses on the case of perfect CSI, but the case of imperfect CSI can be treated similarly by incorporating the channel estimation error model as done in [17].

The rest of the paper is organized as follows. The proposed joint linear design of precoder and decoder in downlink MU-MIMO systems is presented in Section 1, 
whereas the design for uplink scenario is presented in Section 2 . The superiority of the proposed designs over the conventional designs is verified with simulation results in Section 3. Finally, conclusions are given in Section 4 .

\section{JOINT LINEAR PRECODER AND DECODER DESIGN IN DOWNLINK MU-MIMO SYSTEMS}

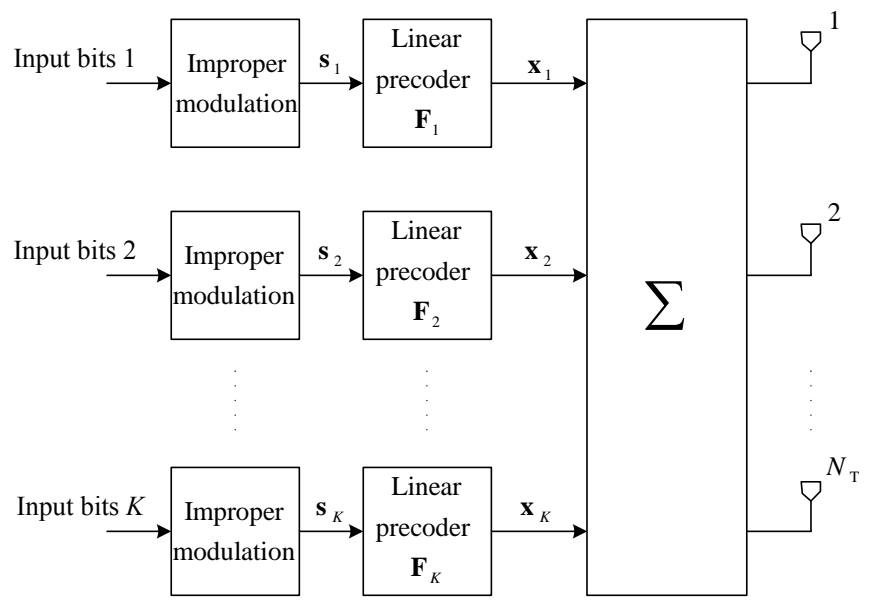

Figure 1. Transmitter in a downlink MU-MIMO system.
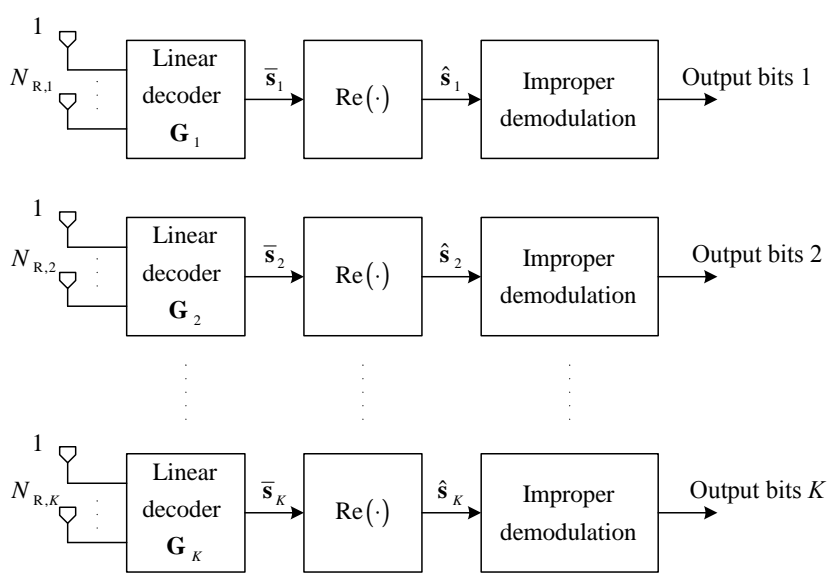

Figure 2. Receivers in a downlink MU-MIMO system.

Figures 1 and 2 illustrate the structures of transmitter and receivers in downlink MU-MIMO systems, respectively. The base-station (BS) has $N_{\mathrm{T}}$ transmit antennas. There are $K$ users in which the $j$ th user is equipped with $N_{R, j}$ receive antennas, $j=1, \ldots, K$. Suppose that user $j$ has $B_{j}$ data streams, represented by $B_{j} \times 1$ vector $\mathbf{s}_{j}$, where $B_{j} \leq \min \left(N_{\mathrm{T}}, N_{\mathrm{R}, j}\right)$. The total number of substreams is therefore $B=\sum_{j=1}^{K} B_{j}$. All the data symbols are assumed to be uncorrelated and have zero mean and unit energy, i.e., $E\left[\mathbf{s}_{j} \mathbf{s}_{j}^{H}\right]=\mathbf{I}_{B, j}$. Furthermore, for improper modulations (such as $M$-ASK), $E\left[\mathbf{s}_{j} \mathbf{s}_{j}^{T}\right] \neq 0$.

The linear precoder for user $j$ at BS is defined as $N_{\mathrm{T}} \times$ $B_{j}$ matrix $\mathbf{F}_{j}$. The output from the $j$ th precoder is $\mathbf{x}_{j}=$ $\mathbf{F}_{j} \mathbf{s}_{j}$. The precoded signals satisfy the following total transmit power constraint:

$$
\sum_{j=1}^{K} E\left[\left\|\mathbf{F}_{j} \mathbf{s}_{j}\right\|^{2}\right]=\sum_{j=1}^{K} \operatorname{Tr}\left(\mathbf{F}_{j} \mathbf{F}_{j}^{H}\right)=P_{\mathrm{T}} .
$$

Precoded signals from all users are simultaneously transmitted across slowly-varying flat Rayleigh fading channels. The downlink channels to user $j$ are collectively represented in matrix $\mathbf{H}_{i}$. The signal received by user $i$ is given by [11], [12],

$$
\mathbf{y}_{i}^{(\mathrm{DL})}=\mathbf{H}_{i}\left[\sum_{j=1}^{K} \mathbf{F}_{j} \mathbf{s}_{j}\right]+\mathbf{n}_{i}^{(\mathrm{DL})},
$$

which is a vector of size $N_{\mathrm{R}, i} \times 1$. The $N_{\mathrm{R}, i} \times 1$ vector $\mathbf{n}_{i}^{(\mathrm{DL})}$ represents spatially and temporally additive white Gaussian noise (AWGN) of zero mean and variance $\left(\sigma_{\mathrm{n}}^{(\mathrm{DL})}\right)^{2}$. The received vector is then fed to the decoder $\mathbf{G}_{i}, i=1, \ldots, K$, which is a $B_{i} \times N_{\mathrm{R}, i}$ matrix. The output of the decoder is:

$$
\mathbf{r}_{i}^{(\mathrm{DL})}=\mathbf{G}_{i} \mathbf{H}_{i}\left[\sum_{j=1}^{K} \mathbf{F}_{j} \mathbf{s}_{j}\right]+\mathbf{G}_{i} \mathbf{n}_{i}^{(\mathrm{DL})} .
$$

The conventional downlink transceiver design is formulated as a problem of minimizing the total mean squared error (TMSE) under the total transmit power constraint specified by (1). The TMSE corresponding to the $i$ th user is

$$
\begin{aligned}
\epsilon_{i}^{(\mathrm{DL})} & =E\left[\left\|\mathbf{r}_{i}^{(\mathrm{DL})}-\mathbf{s}_{i}\right\|^{2}\right] \\
& =E\left[\left\|\mathbf{G}_{i} \mathbf{H}_{i}\left[\sum_{j=1}^{K} \mathbf{F}_{j} \mathbf{s}_{j}\right]+\mathbf{G}_{i} \mathbf{n}_{i}^{(\mathrm{DL})}-\mathbf{s}_{i}\right\|^{2}\right] .
\end{aligned}
$$

For the case of proper modulation $E\left[\mathbf{s}_{j} \mathbf{s}_{j}^{T}\right]=0$ and the conventional transceiver design based on (3) is optimum. However, with improper modulations, the decoding decision is based on only real part of the output. As in [17], the error vector of user $i$ is modified for improper constellations as $\mathbf{e}^{(\mathrm{DL})}=\hat{\mathbf{r}}_{i}^{(\mathrm{DL})}-\mathbf{s}_{i}$, where

$$
\hat{\mathbf{r}}_{i}^{(\mathrm{DL})}=\Re\left(\mathbf{G}_{i} \mathbf{H}_{i}\left[\sum_{j=1}^{K} \mathbf{F}_{j} \mathbf{s}_{j}\right]+\mathbf{G}_{i} \mathbf{n}_{i}^{(\mathrm{DL})}\right) .
$$

With the error vector defined as above, the MSE of the $i$ th user can be computed as:

$$
\begin{aligned}
& E\left[\left\|\mathbf{e}^{(\mathrm{DL})}\right\|^{2}\right]=E\left[\left\|\hat{\mathbf{r}}_{i}^{(\mathrm{DL})}-\mathbf{s}_{i}\right\|^{2}\right] \\
& =E\left[\left\|\Re\left(\mathbf{G}_{i} \mathbf{H}_{i}\left[\sum_{j=1}^{K} \mathbf{F}_{j} \mathbf{s}_{j}\right]+\mathbf{G}_{i} \mathbf{n}_{i}^{(\mathrm{DL})}\right)-\mathbf{s}_{i}\right\|^{2}\right] \\
& =\operatorname{Tr}\left\{E \left\{\left[\alpha+0.5\left(\mathbf{G}_{i} \mathbf{n}_{i}^{(\mathrm{DL})}+\mathbf{G}_{i}^{*}\left(\mathbf{n}_{i}^{(\mathrm{DL})}\right)^{*}\right)-\mathbf{s}_{i}\right]\right.\right. \\
& \left.\left.\left[\beta+0.5\left(\left(\mathbf{n}_{i}^{(\mathrm{DL})}\right)^{H} \mathbf{G}_{i}^{H}+\left(\mathbf{n}_{i}^{(\mathrm{DL})}\right)^{T} \mathbf{G}_{i}^{T}\right)-\mathbf{s}_{i}^{H}\right]\right\}\right\},
\end{aligned}
$$

where

$$
\begin{aligned}
& \alpha=0.5\left(\mathbf{G}_{i} \mathbf{H}_{i}\left[\sum_{j=1}^{K} \mathbf{F}_{j} \mathbf{s}_{j}\right]+\mathbf{G}_{i}^{*} \mathbf{H}_{i}^{*}\left[\sum_{j=1}^{K} \mathbf{F}_{j}^{*} \mathbf{s}_{j}^{*}\right]\right), \\
& \beta=0.5\left(\left[\sum_{j=1}^{K} \mathbf{s}_{j}^{H} \mathbf{F}_{j}^{H}\right] \mathbf{H}_{i}^{H} \mathbf{G}_{i}^{H}+\left[\sum_{j=1}^{K} \mathbf{s}_{j}^{T} \mathbf{F}_{j}^{T}\right] \mathbf{G}_{i}^{T}\right) .
\end{aligned}
$$


From the assumptions on the statistics of the channel, noise and data, one has

$$
\begin{gathered}
E\left[\mathbf{s}_{i} \mathbf{s}_{i}^{H}\right]=E\left[\mathbf{s}_{i} \mathbf{s}_{i}^{T}\right]=\mathbf{I}_{B, i}, \\
E\left[\mathbf{n}_{i}^{(\mathrm{DL})}\left(\mathbf{n}_{i}^{(\mathrm{DL})}\right)^{H}\right]=\left(\sigma_{\mathrm{n}, i}^{(\mathrm{DL})}\right)^{2} \mathbf{I}_{\mathrm{N}_{T}} \\
E\left[\mathbf{n}_{i}^{(\mathrm{DL})}\right]=0, \\
E\left[\mathbf{n}_{i}^{(\mathrm{DL})}\left(\mathbf{n}_{i}^{(\mathrm{DL})}\right)^{T}\right]=0, \\
E\left[\left(\mathbf{n}_{i}^{(\mathrm{DL})}\right)^{*}\left(\mathbf{n}_{i}^{(\mathrm{DL})}\right)^{H}\right]=0 .
\end{gathered}
$$

Using these facts and after some manipulations (4) simplifies to

$$
\begin{aligned}
& E\left[\left\|\mathbf{e}^{(\mathrm{DL})}\right\|^{2}\right]=\operatorname{Tr}\{0.25(\gamma+\delta+\lambda+\zeta) \\
& \quad-0.5\left(\mathbf{G}_{i} \mathbf{H}_{i} \mathbf{F}_{i}+\mathbf{G}_{i}^{*} \mathbf{H}_{i}^{*} \mathbf{F}_{i}^{*}+\mathbf{F}_{i}^{H} \mathbf{H}_{i}^{H} \mathbf{G}_{i}^{H}+\mathbf{F}_{i}^{T} \mathbf{H}_{i}^{T} \mathbf{G}_{i}^{T}\right) \\
& \left.\quad+\mathbf{I}_{B, i}+0.25\left(\sigma_{\mathrm{n}}^{(\mathrm{DL})}\right)^{2}\left(\mathbf{G}_{i} \mathbf{G}_{i}^{H}+\mathbf{G}_{i}^{*} \mathbf{G}_{i}^{T}\right)\right\},
\end{aligned}
$$

where

$$
\begin{aligned}
& \gamma=\mathbf{G}_{i} \mathbf{H}_{i}\left[\sum_{j=1}^{K} \mathbf{F}_{j} \mathbf{F}_{j}^{H}\right] \mathbf{H}_{i}^{H} \mathbf{G}_{i}^{H}, \\
& \delta=\mathbf{G}_{i} \mathbf{H}_{i}\left[\sum_{j=1}^{K} \mathbf{F}_{j} \mathbf{F}_{j}^{T}\right] \mathbf{H}_{i}^{T} \mathbf{G}_{i}^{T}, \\
& \lambda=\mathbf{G}_{i}^{*} \mathbf{H}_{i}^{*}\left[\sum_{j=1}^{K} \mathbf{F}_{j}^{*} \mathbf{F}_{j}^{H}\right] \mathbf{H}_{i}^{H} \mathbf{G}_{i}^{H}, \\
& \zeta=\mathbf{G}_{i}^{*} \mathbf{H}_{i}^{*}\left[\sum_{j=1}^{K} \mathbf{F}_{i}^{*} \mathbf{F}_{i}^{T}\right] \mathbf{H}_{i}^{T} \mathbf{G}_{i}^{T} .
\end{aligned}
$$

The main objective of downlink MU-MIMO transceiver design is to find a pair of precoding matrix, $\left(\mathbf{F}_{i}\right)$, and decoding matrix, $\left(\mathbf{G}_{i}\right)$, to minimize $E\left[\left\|\mathbf{e}^{(\mathrm{DL})}\right\|^{2}\right]$ subject to the total BS transmit power constraint. That is, the improved TMSE design for downlink MU-MIMO systems employing improper modulations is expressed as

$$
\min _{\left\{\left(\mathbf{F}_{j}, \mathbf{G}_{j}\right)\right\}_{j=1}^{K}} E\left[\left\|\mathbf{e}^{(\mathrm{DL})}\right\|^{2}\right] \quad \text { s.t. } \quad \sum_{j=1}^{K} \operatorname{Tr}\left\{\mathbf{F}_{j} \mathbf{F}_{j}^{H}\right\} \leq P_{\mathrm{T}} .
$$

In order to find a solution to problem (8), form the Lagrangian:

$$
\eta^{(\mathrm{DL})}=E\left[\left\|\mathbf{e}^{(\mathrm{DL})}\right\|^{2}\right]+\mu^{(\mathrm{DL})}\left(\left[\sum_{j=1}^{K} \operatorname{Tr}\left\{\mathbf{F}_{j} \mathbf{F}_{j}^{H}\right\}\right]-P_{\mathrm{T}}\right),
$$

where $\mu^{(\mathrm{DL})}$ is the Lagrange multiplier. By substituting (7) into (9) and then taking the derivatives of $\eta^{(\mathrm{DL})}$ with respect to $\mathbf{F}_{i}$ and $\mathbf{G}_{i}$ [18], the associated Karush-KuhnTucker (KKT) conditions can be obtained and given in the following.

First, the value of $\partial \eta^{(\mathrm{DL})} / \partial \mathbf{G}_{z}$ can be found by using the cyclic property of the trace function. Setting $\partial \eta^{(\mathrm{DL})} / \partial \mathbf{G}_{z}=0,1 \leq z \leq K$, and taking the complex conjugates of both sides yields

$$
\Omega+\Psi+\left(\sigma_{\mathrm{n}}^{(\mathrm{DL})}\right)^{2} \mathbf{G}_{z}=2 \mathbf{F}_{z}^{H} \mathbf{H}_{z}^{H}
$$

where

$$
\begin{aligned}
& \Omega=\mathbf{G}_{z} \mathbf{H}_{z}\left[\sum_{j=1}^{K} \mathbf{F}_{j} \mathbf{F}_{j}^{H}\right] \mathbf{H}_{z}^{H}, \\
& \Psi=\mathbf{G}_{z}^{*} \mathbf{H}_{z}^{*}\left[\sum_{j=1}^{K} \mathbf{F}_{j}^{*} \mathbf{F}_{j}^{H}\right] \mathbf{H}_{z}^{H} .
\end{aligned}
$$

Similarly, setting $\partial \eta^{(\mathrm{DL})} / \partial \mathbf{F}_{z}=0$ and taking the complex conjugates of both sides yields

$$
\Theta+\Lambda+2 \mu^{(\mathrm{DL})} \mathbf{F}_{z}=2 \mathbf{H}_{z}^{H} \mathbf{G}_{z}^{H}
$$

where

$$
\begin{aligned}
& \Theta=\left[\sum_{j=1}^{K} \mathbf{H}_{j}^{H} \mathbf{G}_{j}^{H} \mathbf{G}_{j} \mathbf{H}_{j}\right] \mathbf{F}_{z}, \\
& \Lambda=\left[\sum_{j=1}^{K} \mathbf{H}_{j}^{H} \mathbf{G}_{j}^{H} \mathbf{G}_{j}^{*} \mathbf{H}_{j}^{*}\right] \mathbf{F}_{z}^{*} .
\end{aligned}
$$

Next, by post-multiplying both sides of (10) by $\mathbf{G}_{z}^{H}$ and performing $\sum_{z=1}^{K}$ on both sides, one obtains

$$
\begin{array}{r}
\sum_{z=1}^{K}\left\{\Omega \mathbf{G}_{z}^{H}+\Psi \mathbf{G}_{z}^{H}+\left(\sigma_{\mathrm{n}}^{(\mathrm{DL})}\right)^{2} \mathbf{G}_{z} \mathbf{G}_{z}^{H}\right\}= \\
2 \sum_{z=1}^{K} \mathbf{F}_{z}^{H} \mathbf{H}_{z}^{H} \mathbf{G}_{z}^{H} .
\end{array}
$$

In a similar fashion, by pre-multiplying both sides of (11) with $\mathbf{F}_{z}^{H}$ and performing the sum $\sum_{z=1}^{K}$ on both sides, one has

$$
\begin{gathered}
\sum_{z=1}^{K}\left\{\mathbf{F}_{z}^{H} \Theta+\mathbf{F}_{z}^{H} \Lambda+\mathbf{F}_{z}^{H} 2 \mu^{(\mathrm{DL})} \mathbf{F}_{z}\right\}= \\
2 \sum_{z=1}^{K} \mathbf{F}_{z}^{H} \mathbf{H}_{z}^{H} \mathbf{G}_{z}^{H} .
\end{gathered}
$$

It then follows from (12) and (13) that

$$
\begin{aligned}
\sum_{z=1}^{K}\left\{\Omega \mathbf{G}_{z}^{H}+\right. & \left.\Psi \mathbf{G}_{z}^{H}+\left(\sigma_{\mathrm{n}}^{(\mathrm{DL})}\right)^{2} \mathbf{G}_{z} \mathbf{G}_{z}^{H}\right\}= \\
& \sum_{z=1}^{K}\left\{\mathbf{F}_{z}^{H} \Theta+\mathbf{F}_{z}^{H} \Lambda+\mathbf{F}_{z}^{H} 2 \mu^{(\mathrm{DL})} \mathbf{F}_{z}\right\} .
\end{aligned}
$$

The quantity $\mu^{(\mathrm{DL})}$ is obtained by taking the traces of both sides of (14). The result is

$$
\mu^{(\mathrm{DL})}=\frac{\left(\sigma_{\mathrm{n}}^{(\mathrm{DL})}\right)^{2}}{2 P_{\mathrm{T}}} \sum_{z=1}^{K} \operatorname{Tr}\left\{\mathbf{G}_{z} \mathbf{G}_{z}^{H}\right\} .
$$

At this point, as in [16], an iterative procedure can be readily developed to find the solutions of $\mathbf{F}_{z}$ and $\mathbf{G}_{z}$ based on (10), (11) and (15). First, define

$$
\begin{aligned}
\mathbf{G}_{z} & =\mathbf{G}_{z, \operatorname{Re}}+j \mathbf{G}_{z, \mathrm{Im},} \\
\mathbf{H}_{z}\left[\sum_{j=1}^{K} \mathbf{F}_{j} \mathbf{F}_{j}^{H}\right] \mathbf{H}_{z}^{H} & =\mathbf{A}_{z, \operatorname{Re}}^{(\mathrm{DL})}+j \mathbf{A}_{z, \mathrm{Im}}^{(\mathrm{DL})}, \\
\mathbf{H}_{z}^{*}\left[\sum_{j=1}^{K} \mathbf{F}_{j}^{*} \mathbf{F}_{j}^{H}\right] \mathbf{H}_{z}^{H} & =\mathbf{B}_{z, \operatorname{Re}}^{(\mathrm{DL})}+j \mathbf{B}_{z, \mathrm{Im})}^{(\mathrm{DL})} \\
2 \mathbf{F}_{z}^{H} \mathbf{H}_{z}^{H} & =\mathbf{C}_{z, \operatorname{Re}}^{(\mathrm{DL})}+\mathbf{C}_{z, \mathrm{Im}}^{(\mathrm{DL})} .
\end{aligned}
$$




$$
\begin{aligned}
& {\left[\begin{array}{ll}
\mathbf{C}_{z, \operatorname{Re}}^{(\mathrm{DL})} & \mathbf{C}_{z, \operatorname{Im}}^{(\mathrm{DL})}
\end{array}\right]=\left[\begin{array}{ll}
\mathbf{G}_{z, \operatorname{Re}} & \mathbf{G}_{z, \operatorname{Im}}
\end{array}\right]\left[\begin{array}{cc}
\mathbf{A}_{z, \operatorname{Re}}^{(\mathrm{DL})}+\mathbf{B}_{z, \operatorname{Re}}^{(\mathrm{DL})}+\left(\sigma_{\mathrm{n}}^{(\mathrm{DL})}\right)^{2} \mathbf{I}_{N_{\mathrm{R}, z}} & \mathbf{A}_{z, \operatorname{Im})}^{(\mathrm{DL})}+\mathbf{B}_{z, \operatorname{Im}}^{(\mathrm{DL})} \\
\mathbf{B}_{z, \operatorname{Im}}^{(\mathrm{DL})}-\mathbf{A}_{z, \operatorname{Im}}^{(\mathrm{DL})} & \mathbf{A}_{z, \operatorname{Re}}^{(\mathrm{DL})}+\mathbf{B}_{z, \operatorname{Re}}^{(\mathrm{DL})}-\left(\sigma_{\mathrm{n}}^{(\mathrm{DL})}\right)^{2} \mathbf{I}_{N_{\mathrm{R}, z}}
\end{array}\right]} \\
& {\left[\begin{array}{ll}
\mathbf{G}_{z, \operatorname{Re}} & \mathbf{G}_{z, \operatorname{Im}}
\end{array}\right]=\left[\begin{array}{ll}
\mathbf{C}_{z, \operatorname{Re}}^{(\mathrm{DL})} & \mathbf{C}_{z, \operatorname{Im}}^{(\mathrm{DL})}
\end{array}\right]\left[\begin{array}{cc}
\mathbf{A}_{z, \operatorname{Re}}^{(\mathrm{DL})}+\mathbf{B}_{z, \operatorname{Re}}^{(\mathrm{DL})}+\left(\sigma_{\mathrm{n}}^{(\mathrm{DL})}\right)^{2} \mathbf{I}_{N_{\mathrm{R}, z}} & \mathbf{A}_{z, \operatorname{Im}}^{(\mathrm{DL})}+\mathbf{B}_{z, \operatorname{Im}}^{(\mathrm{DL})} \\
\mathbf{B}_{z, \operatorname{Im}}^{(\mathrm{DL})}-\mathbf{A}_{z, \operatorname{Im}}^{(\mathrm{DL})} & \mathbf{A}_{z, \operatorname{Re}}^{(\mathrm{DL})}+\mathbf{B}_{z, \operatorname{Re}}^{(\mathrm{DL})}-\left(\sigma_{\mathrm{n}}^{(\mathrm{DL})}\right)^{2} \mathbf{I}_{N_{\mathrm{R}, z}}
\end{array}\right]^{-1}} \\
& {\left[\begin{array}{l}
\mathbf{R}_{z, \operatorname{Re}}^{(\mathrm{DL})} \\
\mathbf{R}_{z, \operatorname{Im}}^{(\mathrm{DL})}
\end{array}\right]=\left[\begin{array}{cc}
\mathbf{P}_{z, \operatorname{Re}}^{(\mathrm{DL})}+\mathbf{Q}_{z, \operatorname{Re}}^{(\mathrm{DL})}+2 \mu^{(\mathrm{DL})} \mathbf{I}_{N_{\mathrm{T}}} & \mathbf{Q}_{z, \mathrm{Im}}^{(\mathrm{DL})}-\mathbf{P}_{z, \mathrm{Im}}^{(\mathrm{DL})} \\
\mathbf{P}_{z, \operatorname{Im}}^{(\mathrm{DL})}+\mathbf{Q}_{z, \operatorname{Im}}^{(\mathrm{DL})} & \mathbf{P}_{z, \operatorname{Re}}^{(\mathrm{DL})} \mathbf{Q}_{z, \operatorname{Re}}^{(\mathrm{DL})}-2 \mu^{(\mathrm{DL})} \mathbf{I}_{N_{\mathrm{T}}}
\end{array}\right]\left[\begin{array}{l}
\mathbf{F}_{z, \operatorname{Re}} \\
\mathbf{F}_{z, \mathrm{Im}}
\end{array}\right]} \\
& {\left[\begin{array}{c}
\mathbf{F}_{z, \operatorname{Re}} \\
\mathbf{F}_{z, \operatorname{Im}}
\end{array}\right]=\left[\begin{array}{cc}
\mathbf{P}_{z, \operatorname{Re}}^{(\mathrm{DL})}+\mathbf{Q}_{z, \mathrm{Re}}^{(\mathrm{DL})}+2 \mu^{(\mathrm{DL})} \mathbf{I}_{N_{\mathrm{T}}} & \mathbf{Q}_{z, \mathrm{Im}}^{(\mathrm{DL})}-\mathbf{P}_{z, \mathrm{Im}}^{(\mathrm{DL})} \\
\mathbf{P}_{z, \mathrm{Im}}^{(\mathrm{DL})}+\mathbf{Q}_{z, \mathrm{Im}}^{(\mathrm{DL})} & \mathbf{P}_{z, \operatorname{Re}}^{(\mathrm{DL})} \mathbf{Q}_{z, \operatorname{Re}}^{(\mathrm{DL})}-2 \mu^{(\mathrm{DL})} \mathbf{I}_{N_{\mathrm{T}}}
\end{array}\right]^{-1}\left[\begin{array}{l}
\mathbf{R}_{z, \mathrm{Re}}^{(\mathrm{DL})} \\
\mathbf{R}_{z, \operatorname{Im}}^{(\mathrm{DL})}
\end{array}\right]}
\end{aligned}
$$

Then $\mathbf{C}_{z, \operatorname{Re}}^{(\mathrm{DL})}$ and $\mathbf{C}_{z, \mathrm{Im}}^{(\mathrm{DL})}$ can be expressed using (10), in a vector form as given in (20) above, which also implies (21).

Similarly, define

$$
\begin{aligned}
\mathbf{F} z & =\mathbf{F}_{z, \operatorname{Re}}+j \mathbf{F}_{z, \mathrm{Im},} \\
{\left[\sum_{j=1}^{K} \mathbf{H}_{j}^{H} \mathbf{G}_{j}^{H} \mathbf{G}_{j} \mathbf{H}_{j}\right] } & =\mathbf{P}_{z, \operatorname{Re}}^{(\mathrm{DL})}+j \mathbf{P}_{z, \mathrm{Im}}^{(\mathrm{DL})}, \\
{\left[\sum_{j=1}^{K} \mathbf{H}_{j}^{H} \mathbf{G}_{j}^{H} \mathbf{G}_{j}^{*} \mathbf{H}_{j}^{*}\right] } & =\mathbf{Q}_{z, \operatorname{Re}}^{(\mathrm{DL})}+j \mathbf{Q}_{z, \mathrm{Im}}^{(\mathrm{DL})}, \\
2 \mathbf{H}_{z}^{H} \mathbf{G}_{z}^{H} & =\mathbf{R}_{z, \operatorname{Re}}^{(\mathrm{DL})}+\mathbf{R}_{z, \mathrm{Im}}^{(\mathrm{DL})} .
\end{aligned}
$$

Then $\mathbf{R}_{z, \operatorname{Re}}^{(\mathrm{RL})}$ and $\mathbf{R}_{z, \operatorname{Im}}^{(\mathrm{DL})}$ can be expressed using (11) as shown in (22) and equivalently in (23). Based on these expressions, the optimum precoder and decoder can be solved by an iteration procedure as outlined in following algorithm:

1) Initialize $\mathbf{F}_{z}, z=1, \ldots, K$, and $\mathbf{F}_{z}$ by setting the $B_{z} \times B_{z}$ upper sub-matrix of $\mathbf{F}_{z}$ a scaled identity matrix (which satisfies the power constraint with equality), while all the other remaining entries of $\mathbf{F}_{z}$ are zero.

2) For $z=1, \ldots, K$, find the value of $\mathbf{G}_{z}$ using (21).

3) Find the value of $\mu^{(\mathrm{DL})}$ using (15).

4) For $z=1, \ldots, K$, find $\mathbf{F}_{z}$ using (23).

5) If

$$
\operatorname{Tr}\left\{\sum_{z=1}^{K} \mathbf{F}_{z} \mathbf{F}_{z}^{H}\right\}>P_{\mathrm{T}}
$$

scale $\mathbf{F}_{z}$ such that

$$
\operatorname{Tr}\left\{\sum_{z=1}^{K} \mathbf{F}_{\mathbf{z}} \mathbf{F}_{z}^{H}\right\}=P_{\mathrm{T}},
$$

6) If

else go to the next step.

$$
\operatorname{Tr}\left\{\sum_{z=1}^{K}\left(\mathbf{F}_{z}^{i}-\mathbf{F}_{z}^{i-1}\right)\left(\mathbf{F}_{z}^{i}-\mathbf{F}_{z}^{i-1}\right)^{H}\right\}<10^{-4},
$$

then terminate, else go to Step 2.
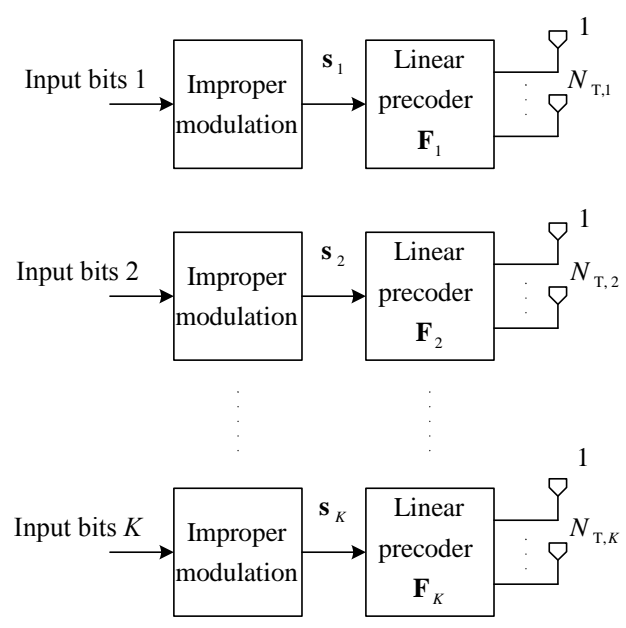

Figure 3. Transmitters in a uplink MU-MIMO system.

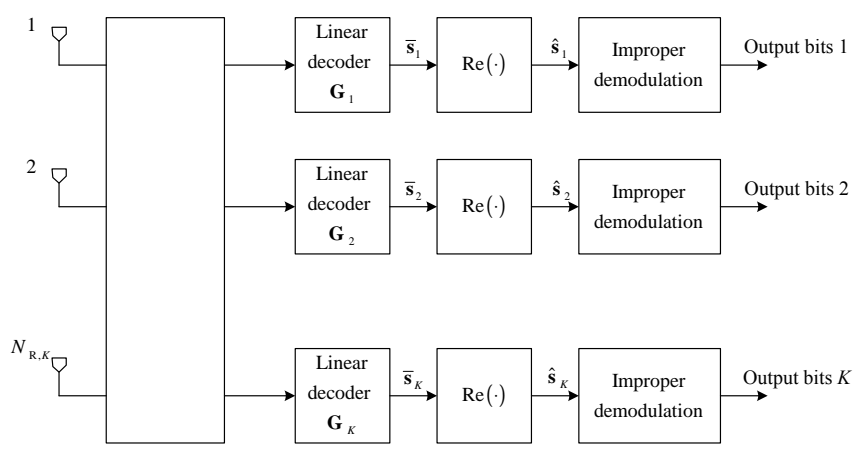

Figure 4. Receiver in a uplink MU-MIMO system.

\section{Transceiver Design in the Uplink of MU-MIMO SYSTEMS}

This section considers uplink MU-MIMO systems where $K$ users (each with $N_{\mathrm{T}, j}$ transmit antennas, $j=$ $1, \ldots, K)$ transmit to a base station equipped with $N_{R}$ antennas. Figures 3 and 4 illustrate the structures of transmitters and receiver in such systems. The linear precoder of user $j$ is denoted by a $N_{\mathrm{T}, j} \times B_{j}$ matrix $\mathbf{F}_{j}$, $j=1, \ldots, K$. The data vector at the output of the $j$ th precoder is represented as $\mathbf{x}_{j}=\mathbf{F}_{j} \mathbf{s}_{j}$. The signal after the precoder satisfies the power constraint in (1). 


$$
\begin{aligned}
& {\left[\begin{array}{ll}
\mathbf{C}_{z, \operatorname{Re}}^{(\mathrm{UL})} & \mathbf{C}_{z, \operatorname{Im}}^{(\mathrm{UL})}
\end{array}\right]=\left[\begin{array}{ll}
\mathbf{G}_{z, \operatorname{Re}} & \mathbf{G}_{z, \operatorname{Im}}
\end{array}\right]\left[\begin{array}{cc}
\mathbf{A}_{z, \operatorname{Re}}^{(\mathrm{UL})}+\mathbf{B}_{z, \operatorname{Re}}^{(\mathrm{UL})}+\left(\sigma_{\mathrm{n}}^{(\mathrm{UL})}\right)^{2} \mathbf{I}_{N_{\mathrm{R}}} & \mathbf{A}_{z, \operatorname{Im})}^{(\mathrm{UL})}+\mathbf{B}_{z, \operatorname{Im}}^{(\mathrm{UL})} \\
\mathbf{B}_{z, \operatorname{Im}}^{(\mathrm{UL})}-\mathbf{A}_{z, \mathrm{Im}}^{(\mathrm{UL})} & \mathbf{A}_{z, \operatorname{Re}}^{(\mathrm{UL})}+\mathbf{B}_{z, \operatorname{Re}}^{(\mathrm{UL})}-\left(\sigma_{\mathrm{n}}^{(\mathrm{UL})}\right)^{2} \mathbf{I}_{N_{\mathrm{R}}}
\end{array}\right]} \\
& {\left[\begin{array}{ll}
\mathbf{G}_{z, \operatorname{Re}} & \mathbf{G}_{z, \operatorname{Im}}
\end{array}\right]=\left[\begin{array}{ll}
\mathbf{C}_{z, \operatorname{Re}}^{(\mathrm{UL})} & \mathbf{C}_{z, \operatorname{Im}}^{(\mathrm{UL})}
\end{array}\right]\left[\begin{array}{cc}
\mathbf{A}_{z, \operatorname{Re}}^{(\mathrm{UL})}+\mathbf{B}_{z, \operatorname{Re}}^{(\mathrm{UL})}+\left(\sigma_{\mathrm{n}}^{(\mathrm{UL})}\right)^{2} \mathbf{I}_{N_{\mathrm{R}}} & \mathbf{A}_{z, \mathrm{Im}}^{(\mathrm{UL})}+\mathbf{B}_{z, \operatorname{Im}}^{(\mathrm{UL})} \\
\mathbf{B}_{z, \operatorname{Im}}^{(\mathrm{UL})}-\mathbf{A}_{z, \operatorname{Im}}^{(\mathrm{UL})} & \mathbf{A}_{z, \operatorname{Re}}^{(\mathrm{UL})}+\mathbf{B}_{z, \operatorname{Re}}^{(\mathrm{UL})}-\left(\sigma_{\mathrm{n}}^{(\mathrm{UL})}\right)^{2} \mathbf{I}_{N_{\mathrm{R}}}
\end{array}\right]^{-1}} \\
& {\left[\begin{array}{l}
\mathbf{R}_{z, \operatorname{Re}}^{(\mathrm{UL})} \\
\mathbf{R}_{z, \mathrm{Im}}^{(\mathrm{UL})}
\end{array}\right]=\left[\begin{array}{cc}
\mathbf{P}_{z, \operatorname{Re}}^{(\mathrm{UL})}+\mathbf{Q}_{z, \operatorname{Re}}^{(\mathrm{UL})}+2 \mu^{(\mathrm{UL})} \mathbf{I}_{N_{\mathrm{T}, \mathrm{z}}} & \mathbf{Q}_{z, \mathrm{Im})}^{(\mathrm{UL})} \mathbf{P}_{z, \mathrm{Im}}^{(\mathrm{UL})} \\
\mathbf{P}_{z, \mathrm{Im}}^{(\mathrm{UL})}+\mathbf{Q}_{z, \mathrm{Im}}^{(\mathrm{UL})} & \mathbf{P}_{z, \operatorname{Re}}^{(\mathrm{UL})}+\mathbf{Q}_{z, \operatorname{Re}}^{(U L)}-2 \mu^{(\mathrm{UL})} \mathbf{I}_{N_{\mathrm{T}, z}}
\end{array}\right]\left[\begin{array}{l}
\mathbf{F}_{z, \operatorname{Re}} \\
\mathbf{F}_{z, \mathrm{Im}}
\end{array}\right]} \\
& {\left[\begin{array}{c}
\mathbf{F}_{z, \operatorname{Re}} \\
\mathbf{F}_{z, \operatorname{Im}}
\end{array}\right]=\left[\begin{array}{cc}
\mathbf{P}_{z, \operatorname{Re}}^{(\mathrm{UL})}+\underset{\mathbf{Q}_{z, \operatorname{Re}}^{(\mathrm{UL})}+2 \mu^{(\mathrm{UL})} \mathbf{I}_{N_{\mathrm{T}, \mathrm{z}}}}{\mathbf{Q}_{z, \mathrm{Im}}^{(\mathrm{UL})}-\mathbf{P}_{z, \mathrm{Im}}^{(\mathrm{UL})}} \\
\mathbf{P}_{z, \mathrm{Im}}^{(\mathrm{UL})}+\mathbf{Q}_{z, \mathrm{Im}}^{(\mathrm{UL})} & \mathbf{P}_{z, \operatorname{Re}}+\mathbf{Q}_{z, \operatorname{Re}}^{(U L)}-2 \mu^{(\mathrm{UL})} \mathbf{I}_{N_{\mathrm{T}, z}}
\end{array}\right]^{-1}\left[\begin{array}{l}
\mathbf{R}_{z, \operatorname{Re}}^{(\mathrm{UL})} \\
\mathbf{R}_{z, \operatorname{Im}}^{(\mathrm{UL})}
\end{array}\right]}
\end{aligned}
$$

At the BS, the received signal vector [11],

$$
\mathbf{y}^{(\mathrm{UL})}=\left[\sum_{j=1}^{K} \mathbf{H}_{j} \mathbf{F}_{j} \mathbf{s}_{j}\right]+\mathbf{n}^{(\mathrm{UL})}
$$

is fed to the decoder $\mathbf{G}_{i}, i=1, \ldots, K$, which is a $B_{i} \times N_{\mathrm{R}}$ matrix. Then the estimation of resultant vector for user $i$ is:

$$
\mathbf{r}_{i}^{(\mathrm{UL})}=\mathbf{G}_{i}\left[\sum_{j=1}^{K} \mathbf{H}_{j} \mathbf{F}_{j} \mathbf{s}_{j}\right]+\mathbf{G}_{i} \mathbf{n}^{(\mathrm{UL})}
$$

where the $N_{\mathrm{R}} \times 1$ vector $\mathbf{n}^{(\mathrm{UL})}$ represents spatially and temporally additive white Gaussian noise (AWGN) of zero mean and variance $\left(\sigma_{\mathrm{n}}^{(\mathrm{UL})}\right)^{2}$. The conventional transceivers are derived by minimizing the following TMSE:

$$
\begin{aligned}
\epsilon_{i}^{(\mathrm{UL})} & =E\left[\left\|\mathbf{r}_{i}^{(\mathrm{UL})}-\mathbf{s}_{i}\right\|^{2}\right] \\
& =E\left[\left\|\mathbf{G}_{i}\left[\sum_{j=1}^{k} \mathbf{H}_{j} \mathbf{F}_{j} \mathbf{s}_{j}\right]+\mathbf{G}_{i} \mathbf{n}^{(\mathrm{UL})}-\mathbf{s}_{i}\right\|^{2}\right] .
\end{aligned}
$$

As in the case of downlink design, with an improper constellation, the error vector is redefined as $\mathbf{e}^{(\mathrm{UL})}=$ $\hat{\mathbf{r}}_{i}^{(\mathrm{UL})}-\mathbf{s}_{i}$, where

$$
\hat{\mathbf{r}}_{i}^{(\mathrm{UL})}=\Re\left(\mathbf{G}_{i}\left[\sum_{j=1}^{K} \mathbf{H}_{j} \mathbf{F}_{j} \mathbf{s}_{j}\right]+\mathbf{G}_{i} \mathbf{n}^{(\mathrm{UL})}\right) .
$$

With the redefined error vector, the TMSE can be computed as follows:

$$
\begin{aligned}
& E\left[\left\|\mathbf{e}^{(\mathrm{UL})}\right\|^{2}\right]=E\left[\left\|\hat{\mathbf{r}}_{i}^{(\mathrm{UL})}-\mathbf{s}_{i}\right\|^{2}\right] \\
& \quad=E\left[\left\|\Re\left(\mathbf{G}_{i}\left[\sum_{j=1}^{K} \mathbf{H}_{j} \mathbf{F}_{j} \mathbf{s}_{j}\right]+\mathbf{G}_{i} \mathbf{n}^{(\mathrm{UL})}\right)-\mathbf{s}_{i}\right\|^{2}\right] .
\end{aligned}
$$

The joint transceiver design for minimum TMSE in the uplink scenario can be expressed as

$$
\min _{\left\{\mathbf{F}_{j}, \mathbf{G}_{j}\right\}_{j=1}^{K}} E\left[\left\|\mathbf{e}^{(\mathrm{UL})}\right\|^{2}\right] \quad \text { s.t. } \quad \operatorname{Tr}\left\{\sum_{j=1}^{K} \mathbf{F}_{j} \mathbf{F}_{j}^{H}\right\} \leq P_{\mathrm{T}} .
$$

As before, the Lagrange multiplier is expressed as:

$$
\mu^{(\mathrm{UL})}=\frac{\left(\sigma_{\mathrm{n}}^{(\mathrm{UL})}\right)^{2}}{2 P_{\mathrm{T}}} \operatorname{Tr}\left\{\sum_{z=1}^{K} \mathbf{G}_{z} \mathbf{G}_{z}^{H}\right\}
$$

The solutions of $\mathbf{F}_{z}$ and $\mathbf{G}_{z}$ can then be found using the same procedure as in Section 1. Specifically, express

$$
\begin{aligned}
\mathbf{G}_{z} & =\mathbf{G}_{z, \operatorname{Re}}+j \mathbf{G}_{z, \mathrm{Im},} \\
{\left[\sum_{j=1}^{K} \mathbf{H}_{j} \mathbf{F}_{j} \mathbf{F}_{j}^{H} \mathbf{H}_{j}^{H}\right] } & =\mathbf{A}_{z, \operatorname{Re}}^{(\mathrm{UL})}+j \mathbf{A}_{z, \mathrm{Im}}^{(\mathrm{UL})}, \\
{\left[\sum_{j=1}^{K} \mathbf{H}_{j}^{*} \mathbf{F}_{j}^{*} \mathbf{F}_{j}^{H} \mathbf{H}_{j}^{H}\right] } & =\mathbf{B}_{z, \mathrm{Re}}^{(\mathrm{UL})}+j \mathbf{B}_{z, \mathrm{Im}}^{(\mathrm{UL})} \\
2 \mathbf{F}_{z}^{H} \mathbf{H}_{z}^{H} & =\mathbf{C}_{z, \mathrm{Re}}^{(\mathrm{UL})}+\mathbf{C}_{z, \mathrm{Im}}^{(\mathrm{UL})} .
\end{aligned}
$$

Then $\mathbf{C}_{z, \operatorname{Re}}^{(\mathrm{UL})}$ and $\mathbf{C}_{z \text { im }}^{(\mathrm{UL})}$ can be written in a vector form as in Equations (28) and (29) at the top of the page.

Similarly, define

$$
\begin{aligned}
\mathbf{F}_{z} & =\mathbf{F}_{z, \operatorname{Re}}+j \mathbf{F}_{z, \mathrm{Im},} \\
\mathbf{H}_{z}^{H}\left[\sum_{j=1}^{K} \mathbf{G}_{j}^{H} \mathbf{G}_{j}\right] \mathbf{H}_{z} & =\mathbf{P}_{z, \operatorname{Re}}^{(\mathrm{UL})}+j \mathbf{P}_{z, \mathrm{Im}}^{(\mathrm{UL})}, \\
\mathbf{H}_{z}^{H}\left[\sum_{j=1}^{K} \mathbf{G}_{j}^{H} \mathbf{G}_{j}^{*}\right] \mathbf{H}_{z}^{*} & =\mathbf{Q}_{z, \mathrm{Re}}^{(\mathrm{UL})}+j \mathbf{Q}_{z, \mathrm{Im}}^{(\mathrm{UL})}, \\
2 \mathbf{H}_{z}^{H} \mathbf{G}_{z}^{H} & =\mathbf{R}_{z, \mathrm{Re}}^{(\mathrm{UL})}+\mathbf{R}_{z, \mathrm{Im}}^{(\mathrm{UL})} .
\end{aligned}
$$

Then $\mathbf{R}_{z, \operatorname{Re}}^{(\mathrm{UL})}$ and $\mathbf{R}_{z \mathrm{Im}}^{(\mathrm{UL})}$ can be expressed in a vector form as in (30) and, equivalently, in (31), at the top of the page. Based on these expressions, the solutions of precoder and decoder can be solved by an iteration procedure as outlined in Section 1.

\section{Numerical Results}

This section presents computer simulation results to demonstrate the performance of the proposed precoding and decoding designs for both uplink and downlink MU-MIMO systems in terms of bit error rate (BER). In particular, the following comparisons are made.

First, performance of the proposed joint precoding/decoding design for a MU-MIMO system employing improper constellation is compared with the novel linear precoding scheme in [16] which is based on the nullspace of channel transmission matrix to decouple multi-user channels. The main purpose of this comparison is to show the benefit in performing the joint precoding/decoding as opposed to precoding only in a MU-MIMO system. 


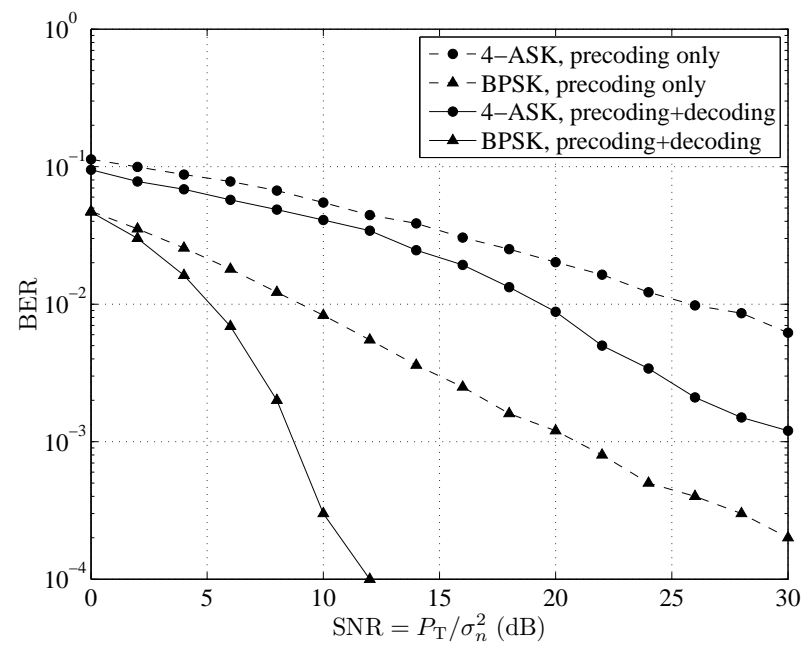

Figure 5. Performance comparison of the precoder design and joint precoder/decoder design in MU-MIMO downlink, for BPSK and 4ASK. $K=3, N_{\mathrm{T}}=6, N_{\mathrm{R}, 1}=N_{\mathrm{R}, 2}=N_{\mathrm{R}, 3}=2, B=2$.

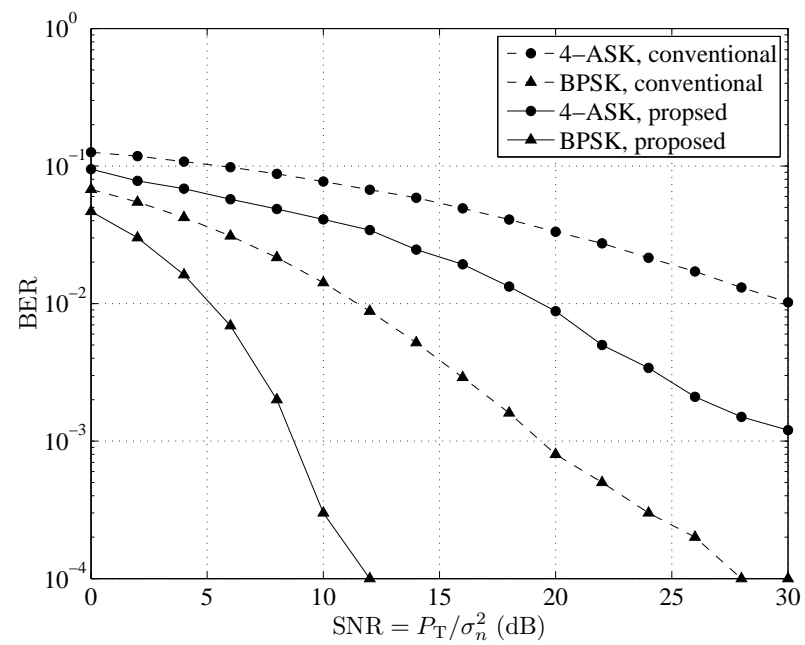

Figure 6. Performance comparison of the conventional downlink transceiver and proposed downlink transceiver, for BPSK, and 4-ASK. $K=3, N_{\mathrm{T}}=6, N_{\mathrm{R}, 1}=N_{\mathrm{R}, 2}=N_{\mathrm{R}, 3}=2, B=2$.

Second, the proposed joint linear transceiver design is compared with the previously-designed joint linear transceiver strategy in [12], but without taking into account the specific property of improper modulations. The comparison is done for both uplink and downlink MU-MIMO systems.

In all the simulation results reported in this section, the number of users is $K=3$. For the downlink design, the number of transmit antennas at the BS is $N_{\mathrm{T}}=6$, while the number of receive antennas for each user is set to be $N_{R, 1}=N_{R, 2}=N_{R, 3}=2$. For the uplink case, the numbers of transmit and receive antennas are set to be $N_{\mathrm{T}, 1}=N_{\mathrm{T}, 2}=N_{\mathrm{T}, 3}=2, N_{\mathrm{R}}=6$ and the number of data streams are $B=2$. For the case of Figure 8, the number of data streams is either $B=1$ or $B=2$ (see the figure legend for details). In all figures, the signal-to-noise ratio is defined as $\mathrm{SNR}=P_{\mathrm{T}} / \sigma_{n}^{2}$ and the BER curves corresponding to user 1 are displayed.

First, Figure 5 compares the performance of the precoding design based on the nullspace of channel trans-

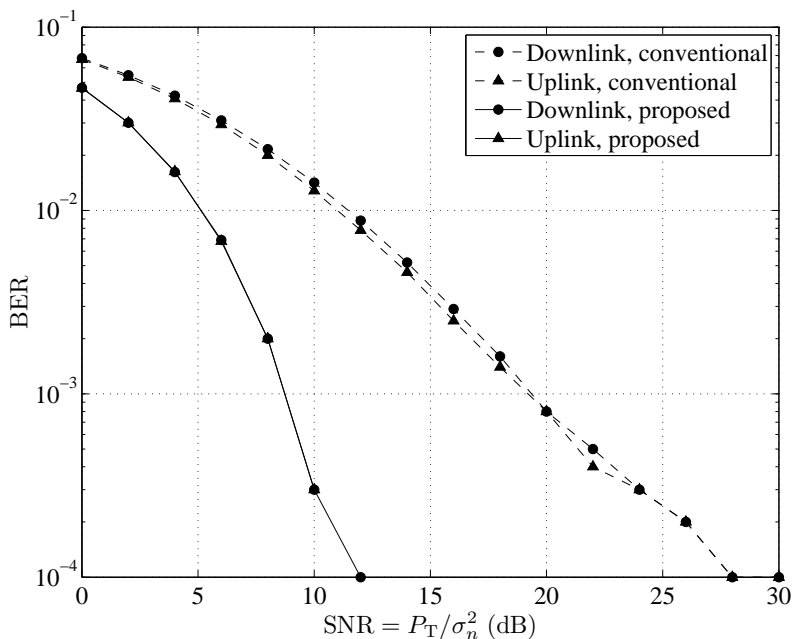

Figure 7. Performance comparison of the downlink and uplink transceivers for BPSK. $K=3, N_{\mathrm{T}}=6, N_{\mathrm{R}, 1}=N_{\mathrm{R}, 2}=N_{\mathrm{R}, 3}=2$, $N_{\mathrm{R}}=6, N_{\mathrm{T}, 1}=N_{\mathrm{T}, 2}=N_{\mathrm{T}, 3}=2 B=2$.

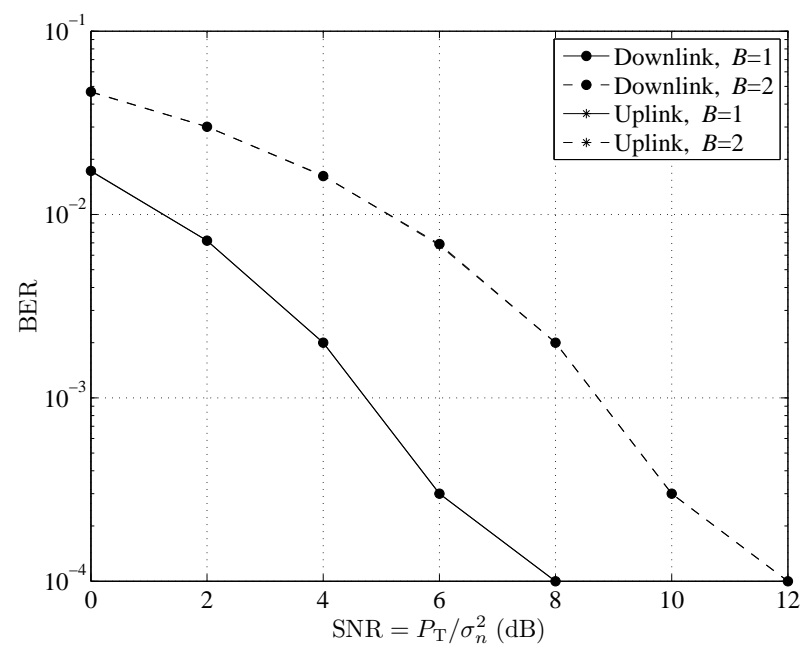

Figure 8. Performance comparison of the proposed downlink and uplink transceivers with $B=1$ and $B=2$ for BPSK. $K=3, N_{\mathrm{T}}=6$, $N_{\mathrm{R}, 1}=N_{\mathrm{R}, 2}=N_{\mathrm{R}, 3}=2$.

mission matrix in downlink MU-MIMO systems [16] with that of the proposed joint precoding and decoding design for BPSK and 4-ASK modulations. It should be emphasized that both the designs under comparison take into account the one-dimensional property of improper modulations. It is clear from the figure that a significant performance improvement is achieved by performing joint precoding and decoding. With the assumption of perfect CSI, it can be seen that the BER performance curves improve exponentially with SNR.

Figure 6 shows performance comparisons of the conventional joint transceiver design for downlink MUMIMO systems in [12] and the proposed joint transceiver design for both BPSK and 4-ASK. As mentioned before, the conventional design does not take into the onedimensional property of improper modulations. As can be seen from the figure, the proposed joint linear precoding/decoding designs leads to a very large performance improvement, especially for BPSK modulation (an SNR improvement of about $15 \mathrm{~dB}$ is observed for BER of $10^{-3}$ ). 
Finally, performance comparison between downlink and uplink transmissions with BPSK is shown in Figure 7 . It can be observed that the BER performance is almost identical for both uplink and downlink scenarios, a similar observation made in [11]. Furthermore, Figure 8 illustrates the performance improvement in both downlink and uplink transmissions when the number of data streams reduces from $B=2$ to $B=1$, i.e., coding gain is achieved by sacrificing the spectral efficiency.

\section{Conclusions}

This paper addressed the joint designs of linear precoding and decoding (transceivers) with improper constellations for both uplink and downlink transmissions of MU-MIMO systems. In both cases, the linear precoder and decoder designs are accomplished with an iterative procedure. Significant performance gains of the proposed designs over other designs in terms of the system's BER was thoroughly demonstrated with simulation results. Though not presented in the paper, the proposed designs can be easily extended for imperfect CSI as done in [17] for SU-MIMO systems.

\section{REFERENCES}

[1] A. J. Paulraj, D. A. Gore, R. U. Nabar and H. Bolcskei, "An overview of MIMO communications - A key to gigabit wireless," Proc. IEEE, vol. 92, pp. 198-218, Feb. 2004.

[2] D. Astely, E. Dahlman, A. Furuskar, Y. Jading, M. Lindstrom and S. Parkvall, "LTE: The evolution of mobile broadband," IEEE Communications Magazine, vol. 47, pp. 44-51, Apr. 2009.

[3] D. P. Palomar, J. M. Cioffi and M. A. Lagunas, "Joint Tx-Rx beamforming design for multicarrier MIMO channels: A unified framework for convex optimization," IEEE Transactions on Signal Processing, vol. 51, pp. 2381-2401, Sep. 2003.

[4] M. Codreanu, A. Tolli, M. Juntti, and M. Latva-aho, "Joint design of Tx-Rx beamformers in MIMO downlink channel," Proc. IEEE Int. Conf. Communications, pp. 4997-5002, June 2007.

[5] S. Serbetli and A. Yener, "Transceiver optimization for multiuser MIMO systems," IEEE Transactions on Signal Processing, vol. 52, pp. 214-226, Jan. 2004.

[6] A. M. Khachan, A. J. Tenenbaum, and R. S. Adve, "Linear processing for the downlink in multiuser MIMO Systems with multiple data streams," Proc. IEEE Int. Conf. Communications, vol. 9, pp. 4113-4118, June 2006.

[7] M. Schubert, Shuying Shi, E. A. Jorswieck, H. Boche, "Downlink sum-MSE transceiver optimization for linear multi-user MIMO systems," Proc. 39 Asilomar Conference on Signals, Systems and Computers., pp. 1424-1428, Nov. 2005.

[8] A. J. Tenenbaum and R. S. Adve, "Joint multiuser transmitreceive optimization using linear processing," Proc. IEEE Int. Conf. Communications, vol. 1, pp. 588-592, June 2004.

[9] S. Serbetli and A. Yener, 'MMSE transmitter design for correlated MIMO systems with imperfect channel estimates: Power allocation trade-offs," IEEE Transactions on Wireless Commnunications, vol. 5, pp. 2295-2304, Aug. 2006.

[10] Minhua Ding and S. D. Blostein, "Joint optimization for multiuser MIMO uplink systems with imperfect CSI," Proc. 24th Biennial Symp. Comm., pp. 191-195, June 2008.

[11] Minhua Ding and S. D. Blostein, "Relation between joint optimizations for multiuser MIMO uplink and downlink with imperfect CSI," Proc. Int. Conf. Acoustics, Speech, and Signal Processing (ICASSP), pp. 3149-3152, Apr. 2008.

[12] Wang Li, Yang Ke, Hu Han-ying and Cui Wei-jia, "Robust joint linear transceiver design for MU-MIMO with imperfect CSI," Proc. GMC, pp. 1-6, Oct. 2010.
[13] T. Yoo and A. Goldsmith, "Capacity and power allocation for fading MIMO channels with channel estimation error," IEEE Transactions on Information Theory, vol. 52, pp. 2203-2214, May 2006.

[14] L. Musavian, M. R.Nakhai, M. Dohler and A. H. Aghvami, "Effect of channel Uncertainty on the Mutual Information of MIMO Fading Channels," IEEE Transactions on Vehicular Technology, vol. 56, pp. 2798-2806, Sep. 2007.

[15] O. Simeone, U. Spagnolini and Y. Bar-Ness "Linear and nonlinear precoding/decoding for MIMO systems using the fading correlation at the transmitter," Proc. 4th IEEE Workshop on SPAWC, pp. 6-10, June 2003.

[16] Pei Xiao and M. Sellathurai, "Improved linear transmit processing for single-user and multi-user MIMO communications systems," IEEE Transactions on Signal Processing, vol. 58, pp. 17681779, Mar. 2010.

[17] M. Raja, P. Muthuchidambaranathan, Ha Nguyen "Transceiver design for MIMO systems with improper modulations," Wireless Personal Communications., Springer Netherlands, pp. 1-16, 2011 http://dx.doi.org/10.1007/s11277-011-0451-z.

[18] G. John Proakis, Digital Communications. McGraw-Hill, 2001.

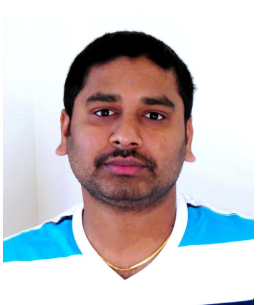

M. Raja received his B.Eng. Degree in Electronics and Communication Engineering from Anna University, Chennai, India, in 2005, and the M.Eng. Degree in Digital Communication and Networking from Anna University, Chennai, India, in 2007. Currently he is pursuing his Ph.D. in Wireless Communication in the Department of Electronics and Communication Engineering, National Institute of Technology (NIT), Tiruchirappalli, India. He was a recipient of Canadian Commonwealth Scholarship Award-2010 for Graduate Student Exchange Program in the Department of Electrical and Computer Engineering, University of Saskatchewan, Saskatoon, SK, Canada and also he is a Visiting Scholar in the Department of Electrical and Computer Engineering, University of Saskatchewan, Saskatoon, SK, Canada during January 2012-June 2012. His research interests include orthogonal frequency division multiplexing (OFDM), multiple-input and multiple-output (MIMO) systems, diversity and beamforming techniques, and channel estimation. He published his research papers in refereed international journals, and international and national conferences.

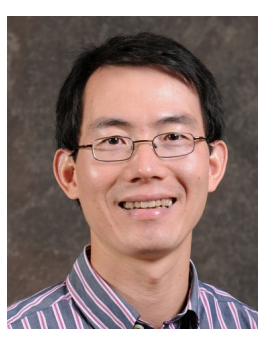

Ha H. Nguyen received the B.Eng. degree from Hanoi University of Technology, Hanoi, Vietnam, in 1995, the M.Eng. degree from the Asian Institute of Technology, Bangkok, Thailand, in 1997, and the Ph.D. degree from the University of Manitoba, Winnipeg, MB, Canada, in 2001, all in electrical engineering. He joined the Department of Electrical Engineering, University of Saskatchewan, Saskatoon, SK, Canada, in 2001, and become a full Professor in 2007. He holds adjunct appointments at the Department of Electrical and Computer Engineering University of Manitoba, Winnipeg, MB, Canada, and TRLabs, Saskatoon. He was a Senior Visiting Fellow in the School of Electrical Engineering and Telecommunications, University of New South Wales, Sydney, Australia, during October 2007 to June 2008. His research interests include digital communications, spread-spectrum systems, and error-control coding. He is a coauthor (with E. Shwedyk) of A First Course in Digital Communications (Cambridge, U.K.: Cambridge University Press, 2009). Dr. Nguyen was an Associate Editor for the IEEE Transactions on Wireless Communications during 2007-2011. He currently serves as an Associate Editor for the IEEE Transactions on Vehicular Technology and the IEEE Wireless Communications Letters. He was a Co-chair for the Multiple Antenna Systems and Space-Time Processing Track, IEEE Vehicular Technology Conferences (Fall 2010, Ottawa, ON, Canada and Fall 2012, Quebec, OC, Canada). He is a Registered Member of the Association of Professional Engineers and Geoscientists of Saskatchewan (APEGS). 


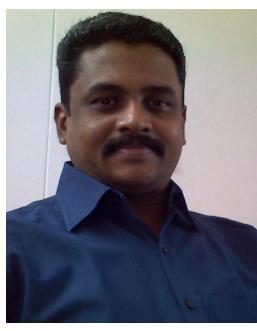

P. Muthuchidambaranathan received his B.Eng. Degree in Electronics and Communication Engineering from Government College of Technology, Coimbatore, India, in 1992, the M.Eng. Degree in Microwave and Optical Engineering, from A.C. College of Engineering and Technology, Karaikudi, India, in 1994. He obtained his Ph.D. degree in optical communication from the National Institute of Technology (NIT), Tiruchirappalli, India in 2009. He is currently working as an Associate Professor in the Department of Electronics and Communication Engineering, National Institute of Technology (NIT), Tiruchirappalli, India. His research interests include wireless communications, and optical communications. He published his research papers in refereed international journals, and international and national conferences. He is an author of the textbook "Wireless Communications" (published by Prentice Hall of India). 